\title{
MULTIPLIERS OF FAMILIES OF CAUCHY-STIELTJES TRANSFORMS
}

\author{
R. A. HIBSCHWEILER AND T. H. MACGREGOR \\ This paper is dedicated to Glenn Schober
}

\begin{abstract}
For $\alpha>0$ let $\mathscr{F}$ denote the class of functions defined for $|z|<1$ by integrating $1 /(1-x z)^{\alpha}$ against a complex measure on $|x|=1$. A function $g$ holomorphic in $|z|<1$ is a multiplier of $\mathscr{F}_{\alpha}$ if $f \in \mathscr{F}_{\alpha}$ implies $g f \in \mathscr{F}_{\alpha}$. The class of all such multipliers is denoted by $\mathscr{K}_{\alpha}$. Various properties of $\mathscr{M}_{\alpha}$ are studied in this paper. For example, it is proven that $\alpha<\beta$ implies $\mathscr{M}_{\alpha} \subset \mathscr{M}_{\beta}$, and also that $\mathscr{M}_{\alpha} \subset H^{\infty}$. Examples are given of bounded functions which are not multipliers. A new proof is given of a theorem of Vinogradov which asserts that if $f^{\prime}$ is in the Hardy class $H^{1}$, then $f \in \mathscr{M}_{1}$. Also the theorem is improved to $f^{\prime} \in H^{1}$ implies $f \in \mathscr{M}_{\alpha}$, for all $\alpha>0$. Finally, let $\alpha>0$ and let $f$ be holomorphic in $|z|<1$. It is known that $f$ is bounded if and only if its Cesàro sums are uniformly bounded in $|z| \leq 1$. This result is generalized using suitable polynomials defined for $\alpha>0$.
\end{abstract}

Let $\Delta=\{z:|z|<1\}$ and $\Gamma=\{z:|z|=1\}$, and let $\mathscr{M}$ denote the set of complex-valued Borel measures on $\Gamma$. For $\alpha>0$, let $\mathscr{F}_{\alpha}$ denote the family of functions $f$ for which there exists $\mu \in \mathscr{M}$ such that

$$
f(z)=\int_{\Gamma} \frac{1}{(1-x z)^{\alpha}} d \mu(x), \quad|z|<1 .
$$

Here we choose the branch of $1 /(1-z)^{\alpha}$ which equals 1 when $z=0$.

This class of functions has been studied extensively in the case $\alpha=1[1,7$, $8,10,15,16]$. More recently, the families $\mathscr{F}_{\alpha}(\alpha \neq 1)$ were introduced in [13]. Closure properties of the families $\mathscr{F}_{\alpha}$ were studied by the present authors in [9].

The following two results were proven in [13], and will be useful here.

Theorem A. For $\alpha>0, f \in \mathscr{F}_{\alpha}$ if and only if $f^{\prime} \in \mathscr{F}_{\alpha+1}$.

Theorem B. If $f \in \mathscr{F}_{\alpha}$ and $g \in \mathscr{F}_{\beta}$, then $f g \in \mathscr{F}_{\alpha+\beta}$.

For $f \in \mathscr{F}_{\alpha}$, let

$$
\|f\|_{\mathscr{F}_{a}}=\inf \{\|\mu\|: \mu \in \mathscr{M} \text { such that (1) holds }\} .
$$

With this norm, $\mathscr{F}_{\alpha}$ is a Banach space. As an example, suppose that $f \in \mathscr{F}$, $\mu$ is a positive measure, and (1) holds. Then $\|f\|_{\mathscr{F}_{\alpha}}=\|\mu\|$. In the case $\alpha=1$,

Received by the editors February 27, 1990.

1980 Mathematics Subject Classification (1985 Revision). Primary 30E20.

Key words and phrases. Cauchy-Stieltjes transforms, complex measures, multipliers. 
this was first observed by P. Bourdon and J. A. Cima, who showed in [1] that if $\nu \in \mathscr{M}$ is any other representing measure for $f$, then

$$
\|\mu\|=\mu(\Gamma)=f(0)=\int_{\Gamma} 1 d \nu(x) \leq\|\nu\| .
$$

We note that by an easy argument, the infimum in (2) is actually attained.

Let $\left\{f_{n}: n=1,2, \ldots\right\}$ be a sequence of functions in $\mathscr{F}_{\alpha}$ and suppose that $f_{n} \rightarrow f$ in the norm (2). It is easy to show that this implies that $f_{n} \rightarrow f$ uniformly on compact sets. To see that the converse is false in the case $\alpha=1$, let $f_{n}(z)=z^{n}$ for $|z|<1$. Then $f_{n}$ converges uniformly on compact sets to the function $f(z)=0$. On the other hand, suppose that $\mu_{n} \in \mathscr{M}$ is any measure representing $f_{n}$. Then since

$$
z^{n}=\int_{\Gamma} \frac{1}{1-x z} d \mu_{n}(x)
$$

it follows that

$$
1=\int_{\Gamma} x^{n} d \mu_{n}(x) \leq \int_{\Gamma} 1 d\left|\mu_{n}\right|(x)=\left\|\mu_{n}\right\| .
$$

This shows that for each $n,\left\|f_{n}\right\|_{\mathscr{F}_{1}} \geq 1$, so that the sequence $f_{n}$ does not converge to $f$ in norm. In the case $\alpha \neq 1$, a similar example can be constructed.

Definition. Suppose that $f$ is holomorphic in $\Delta$. Then $f$ is called a multiplier of $\mathscr{F}_{\alpha}$ if $g \in \mathscr{F}_{\alpha} \Rightarrow f g \in \mathscr{F}_{\alpha}$.

The family of all such multipliers is denoted by $\mathscr{M}_{\alpha}$.

Suppose that $f \in \mathscr{M}_{\alpha}$ for some $\alpha>0$. An application of the Closed Graph Theorem shows that the map $\Lambda: \mathscr{F}_{\alpha} \rightarrow \mathscr{F}_{\alpha}$ defined by $\Lambda(g)=f g$ is continuous. Equivalently, $\Lambda$ is a bounded operator on $\mathscr{F}_{\alpha}$, so that

$$
\sup \left\{\|f g\|_{\mathscr{F}_{\alpha}}: g \in \mathscr{F}_{\alpha},\|g\|_{\mathscr{F}_{\alpha}} \leq 1\right\}<\infty .
$$

This last quantity will be denoted by $\|f\|_{\mathscr{M}_{\alpha}}$, and with this norm $\mathscr{M}_{\alpha}$ is itself a Banach space.

This paper is concerned with the multiplier families $\mathscr{M}_{\alpha}$. The family $\mathscr{M}_{1}$ has been studied in [10], [15], and [16], and various properties of $\mathscr{M}_{1}$ which were developed there will be generalized to $\mathscr{M}_{\alpha}$ for $\alpha \neq 1$. For example, S. A. Vinogradov [16] has shown that if $f^{\prime}$ is in the Hardy space $H^{1}$, then $f \in \mathscr{M}_{1}$. We give a new proof of this result, and show that if $f^{\prime} \in H^{1}$, then $f \in \mathscr{M}_{\alpha}$, for every $\alpha>0$. Also we show that if $f \in \mathscr{M}_{\alpha}$, then $f$ is bounded, and that $f$ has a number of other properties. Examples are given of bounded functions which are not in any $\mathscr{M}_{\alpha}$ for $\alpha>0$.

Finally, suppose that $f$ is holomorphic in $\Delta$, and let $f(z)=\sum_{n=0}^{\infty} a_{n} z^{n}$. Let

$$
\sigma_{n}(z)=\sum_{j=0}^{n} \frac{n-j+1}{n+1} a_{j} z^{j} .
$$

It is a classical result that $f$ is bounded if and only if the Cesàro sums $\sigma_{n}(z)$ are uniformly bounded for $|z| \leq 1$, and that in this case $\left\|\sigma_{n}\right\|_{H^{\infty}} \leq\|f\|_{H^{\infty}}$, 
$n=0,1, \ldots$. This result is generalized here where $\sigma_{n}$ is replaced by suitable polynomials depending on $\alpha>0$.

2

In this section various properties of the families $\mathscr{M}_{\alpha}$ are studied. The following lemma will be useful.

Lemma 2.1. Let $f$ be holomorphic in $\Delta$, and let $\alpha>0$. Then $f \in \mathscr{M}_{\alpha}$ if and only if $f(z) /(1-x z)^{\alpha} \in \mathscr{F}_{\alpha}$ for every $x$ with $|x|=1$ and there exists a constant $M$ such that $\left\|f(z) /(1-x z)^{\alpha}\right\|_{\mathscr{F}_{\alpha}} \leq M$ for $|x|=1$.

Proof. First suppose that $f \in \mathscr{M}_{\alpha}$. Then multiplication by $f$ is a bounded operator on $\mathscr{F}_{\alpha}$, and there is a constant $M$ such that

$$
\|f g\|_{\mathscr{F}_{\alpha}} \leq M\|g\|_{\mathscr{F}_{\alpha}}
$$

for all $g \in \mathscr{F}_{\alpha}$. In particular, (3) holds for all functions of the form $g(z)$ $=1 /(1-x z)^{\alpha}$, where $|x|=1$. Since $\left\|1 /(1-x z)^{\alpha}\right\|_{\mathscr{F}_{\alpha}}=1$, this implies that $\left\|f(z) /(1-x z)^{\alpha}\right\|_{\mathscr{F}_{\alpha}} \leq M$ for all $|x|=1$.

For the converse, let $g \in \mathscr{F}_{\alpha}$. Then for some $\mu \in \mathscr{M}$,

$$
g(z)=\int_{\Gamma} \frac{1}{(1-x z)^{\alpha}} d \mu(x) .
$$

To show that $f g \in \mathscr{F}_{\alpha}$, it is enough to consider the case in which $\mu$ is a probability measure. Then $g$ is the limit in the topology of uniform convergence on compact subsets of $\Delta$ of functions of the form

$$
h(z)=\sum_{k=1}^{n} \mu_{k} \frac{1}{\left(1-x_{k} z\right)^{\alpha}}
$$

where $\mu_{k} \geq 0, \sum_{k=1}^{n} \mu_{k}=1,\left|x_{k}\right|=1$, and $n$ is a natural number.

For such a function $h$,

$$
f(z) h(z)=\sum_{k=1}^{n} \mu_{k} \frac{f(z)}{\left(1-x_{k} z\right)^{\alpha}} .
$$

By the assumption, there is a measure $\nu_{k} \in \mathscr{M}$ with $\left\|\nu_{k}\right\| \leq M$ such that

$$
\frac{f(z)}{\left(1-x_{k} z\right)^{\alpha}}=\int_{\Gamma} \frac{1}{(1-x z)^{\alpha}} d \nu_{k}(x) \text {. }
$$

Letting $\lambda=\sum_{k=1}^{n} \mu_{k} \nu_{k}$, (4) can be written as

$$
f(z) h(z)=\int_{\Gamma} \frac{1}{(1-x z)^{\alpha}} d \lambda(x)
$$

where $\lambda \in \mathscr{M}$ and $\|\lambda\| \leq \sum_{k=1}^{n} \mu_{k}\left\|\nu_{k}\right\| \leq M \sum_{k=1}^{n} \mu_{k}=M$.

Since $\{\lambda \in \mathscr{M}:\|\lambda\| \leq M\}$ is compact, an argument using subsequences now yields a measure $\sigma \in \mathscr{M}$ with $\|\sigma\| \leq M$ and $f(z) g(z)=\int_{\Gamma} 1 /(1-x z)^{\alpha} d \sigma(x)$. Therefore $f g \in \mathscr{F}_{\alpha}$, and $f \in \mathscr{M}_{\alpha}$. 
Theorem 2.2. If $0<\alpha<\beta$, then $\mathscr{M}_{\alpha} \subset \mathscr{M}_{\beta}$.

Proof. Let $f \in \mathscr{M}_{\alpha}$. By 2.1 , it is enough to show that $f(z) /(1-x z)^{\beta} \in \mathscr{F}_{\beta}$ for every $x$ with $|x|=1$, and to show that there is a constant $N$ such that $\left\|f(z) /(1-x z)^{\beta}\right\|_{\mathscr{F}_{\beta}} \leq N$, for $|x|=1$.

Since $f \in \mathscr{M}_{\alpha}$, the lemma implies that there is a constant $M$ with

$$
\left\|f(z) /(1-x z)^{\alpha}\right\|_{\mathscr{F}_{\alpha}} \leq M, \quad \text { for }|x|=1 .
$$

Equivalently, for any $x$ with $|x|=1$, there is a measure $\mu_{x} \in \mathscr{M}$ such that

$$
\frac{f(z)}{(1-x z)^{\alpha}}=\int_{\Gamma} \frac{1}{(1-y z)^{\alpha}} d \mu_{x}(y)
$$

and $\left\|\mu_{x}\right\| \leq M$.

Since

(5) yields that

$$
\frac{f(z)}{(1-x z)^{\beta}}=\frac{f(z)}{(1-x z)^{\alpha}} \frac{1}{(1-x z)^{\beta-\alpha}}
$$

$$
\begin{aligned}
\frac{f(z)}{(1-x z)^{\beta}} & =\left\{\int_{\Gamma} \frac{1}{(1-y z)^{\alpha}} d \mu_{x}(y)\right\} \frac{1}{(1-x z)^{\beta-\alpha}} \\
& =\int_{\Gamma} \frac{1}{(1-y z)^{\alpha}} \frac{1}{(1-x z)^{\beta-\alpha}} d u_{x}(y) .
\end{aligned}
$$

For every $x$ and $y$ with $|x|=|y|=1$, there is a probability measure $\nu_{x, y}$ such that

$$
\frac{1}{(1-y z)^{\alpha}} \frac{1}{(1-x z)^{\beta-\alpha}}=\int_{\Gamma} \frac{1}{(1-w z)^{\beta}} d \nu_{x, y}(w) \quad[2, \text { p. 415]. }
$$

Therefore,

$$
\frac{f(z)}{(1-x z)^{\beta}}=\int_{\Gamma} \int_{\Gamma} \frac{1}{(1-w z)^{\beta}} d \nu_{x, y}(w) d \mu_{x}(y) .
$$

Because $\left\|\nu_{x, y}\right\| \leq 1$ and $\left\|\mu_{x}\right\| \leq M$, an argument as in the proof of Lemma 2.1 shows that there is a measure $\lambda \in \mathscr{M}$ with $\|\lambda\| \leq M$ and such that

$$
\frac{f(z)}{(1-x z)^{\beta}}=\int_{\Gamma} \frac{1}{(1-s z)^{\beta}} d \lambda(s) \text {. }
$$

This shows that $f(z) /(1-x z)^{\beta} \in \mathscr{F}_{\beta}$, and that $\left\|f(z) /(1-x z)^{\beta}\right\|_{\mathscr{F}_{\beta}} \leq M$.

Next we obtain several properties of functions in $\mathscr{M}_{\alpha}$. First it is shown that such functions are bounded.

Theorem 2.3. Let $\alpha>0$ and let $f \in \mathscr{M}_{\alpha}$. Then $f \in H^{\infty}$, and $\|f\|_{H^{\infty}} \leq\|f\|_{\mathscr{M}_{\alpha}}$. Proof. Let $M$ be a constant with $\|f\|_{\mathscr{M}_{\alpha}}<M$. Let $z_{0}=r e^{i \theta} \quad(0 \leq r<1)$ and let $x=e^{-i \theta}$.

Since $f \in \mathscr{M}_{\alpha}$, there is a measure $\mu_{x} \in \mathscr{M}$ with $\left\|\mu_{x}\right\|<M$ and such that

$$
\frac{f(z)}{(1-x z)^{\alpha}}=\int_{\Gamma} \frac{1}{(1-y z)^{\alpha}} d \mu_{x}(y) \text {. }
$$

It follows that

$$
f(z)=\int_{\Gamma}\left(\frac{1-x z}{1-y z}\right)^{\alpha} d \mu_{x}(y)
$$


Letting $z=z_{0}$ in (6) yields

$$
\left|f\left(r e^{i \theta}\right)\right|=\left|\int_{\Gamma}\left(\frac{1-r}{1-r \bar{x} y}\right)^{\alpha} d \mu_{x}(y)\right| \leq \int_{\Gamma} d\left|\mu_{x}\right|(y)<M .
$$

Since (7) holds for all $r$ and $\theta$, it follows that $f \in H^{\infty}$ and $\|f\|_{H^{\infty}}<M$, for every $M$ with $M>\|f\|_{\mathscr{M}_{\alpha}}$. Therefore, $\|f\|_{H^{\infty}} \leq\|f\|_{\mathscr{M}_{\alpha}}$.

Theorem 2.4. Let $\alpha>0$, and let $f \in \mathscr{M}_{\alpha}$. Then $f \in \mathscr{F}_{\alpha}$, and $\|f\|_{\mathscr{F}_{\alpha}} \leq\|f\|_{\mathscr{M}_{\alpha}}$. Proof. Let $I(z)=1$ for $|z|<1$. Since

$$
I(z)=\int_{\Gamma} \frac{1}{(1-x z)^{\alpha}} d m(x)
$$

where $m$ denotes normalized Lebesgue measure, $I \in \mathscr{F}_{\alpha}$. Also, since $m$ is a positive measure, the remark in $\S 1$ shows that

$$
\|I\|_{\mathscr{F}_{\alpha}}=\|m\|=1 \text {. }
$$

Since $f \in \mathscr{M}_{\alpha}$ and $I \in \mathscr{F}_{\alpha}$, it follows that $f=f I \in \mathscr{F}_{\alpha}$. Also, since

$$
\|f\|_{\mathscr{F}_{\alpha}}=\|f I\|_{\mathscr{F}_{\alpha}} \leq\|f\|_{\mathscr{M}_{\alpha}}\|I\|_{\mathscr{F}_{\alpha}}
$$

(8) implies that

$$
\|f\|_{\mathscr{F}_{\alpha}} \leq\|f\|_{\mathscr{K}_{\alpha}} \text {. }
$$

We note that the inequality (9) is sharp, because $I \in \mathscr{M}_{\alpha}$ and $\|I\|_{\mathscr{F}_{\alpha}}=1$. As an application of Theorem 2.4 , let

$$
\frac{1}{(1-z)^{\alpha}}=\sum_{n=0}^{\infty} A_{n}(\alpha) z^{n} \quad(|z|<1) \text {, }
$$

and suppose that $f \in \mathscr{M}_{\alpha}$ where $f(z)=\sum_{n=0}^{\infty} a_{n} z^{n} \quad(|z|<1)$. The theorem asserts that for some $\mu \in \mathscr{M}$,

$$
f(z)=\int_{\Gamma} \frac{1}{(1-x z)^{\alpha}} d \mu(x) .
$$

Equations (10) and (11) imply that

$$
a_{n}=A_{n}(\alpha) \int_{\Gamma} x^{n} d \mu(x) .
$$

Since $A_{n}(\alpha)=O\left(n^{\alpha-1}\right)$, and since $\left|\int_{\Gamma} x^{n} d \mu(x)\right| \leq\|\mu\|$, this shows that the coefficients of $f$ obey $\left|a_{n}\right|=O\left(n^{\alpha-1}\right)$.

In the case $0<\alpha<1$, this coefficient estimate provides additional information on functions in $\mathscr{M}_{\alpha}$. Suppose that $f$ is holomorphic in $\Delta$, and that $f(z)=\sum_{n=0}^{\infty} a_{n} z^{n}$. In [16] it was shown that if $\sum_{n=0}^{\infty}\left|a_{n}\right| \log (n+2)<\infty$, then $f \in \mathscr{M}_{1}$. In particular, the function $f(z)=\sum_{n=0}^{\infty}\left(1 / n^{3}\right) z^{2^{n}}$ is in $\mathscr{M}_{1}$, but for $m=2^{n}, a_{m} \neq O\left(m^{\alpha-1}\right)$, for each $\alpha \quad(0<\alpha<1)$. This shows that $f \notin \mathscr{M}_{\alpha}$ for $\alpha<1$. The first author and E. A. Nordgren have shown that $\mathscr{M}_{1} \neq \mathscr{M}_{2}$, and also that for $0<\alpha<\beta<1, \mathscr{M}_{\alpha} \neq \mathscr{M}_{\beta}$. It is an open question to determine if $\mathscr{M}_{\alpha} \neq \mathscr{M}_{\beta}$ for all $\alpha \neq \beta$.

It was shown in [9] that $\mathscr{F}_{\alpha}$ is closed under composition with disk automorphisms $z \rightarrow(z+\xi) /(1+\bar{\xi} z)$, where $|\xi|<1$. This will be used in the proof of the next theorem, which asserts the same result for $\mathscr{M}_{\alpha}$. 
Theorem 2.5. Let $\alpha>0$. If $f \in \mathscr{M}_{\alpha},|\xi|<1$, and $g(z)=f((z+\xi) /(1+\bar{\xi} z))$, then $g \in \mathscr{M}_{\alpha}$.

Proof. Let $h \in \mathscr{F}_{\alpha}$, and let $k(z)=h((z-\xi) /(1-\bar{\xi} z))$. Since the map $w=$ $(z-\xi) /\left(1-\bar{\xi}_{z}\right)$ is an automorphism of $\Delta$, the result in [9] quoted above shows that $k \in \mathscr{F}_{\alpha}$. Since $f \in \mathscr{M}_{\alpha}$, it follows that $m=f k \in \mathscr{F}_{\alpha}$. A second application of the result in [9] implies that $m((z+\xi) /(1+\bar{\xi} z)) \in \mathscr{F}_{\alpha}$. Since

$$
m\left(\frac{z+\xi}{1+\bar{\xi} z}\right)=f\left(\frac{z+\xi}{1+\bar{\xi} z}\right) k\left(\frac{z+\xi}{1+\bar{\xi} z}\right)=g(z) h(z),
$$

this shows that $g \in \mathscr{M}_{\alpha}$.

The following theorem generalizes a result in [16], which showed that if $f \in \mathscr{M}_{1}$, then $f$ has finite radial variation in every direction.

Theorem 2.6. For each $\alpha>0$ there is a constant $A_{\alpha}$ such that if $f \in \mathscr{M}_{\alpha}$, then the radial variation of $f$ in the direction $\theta$ obeys $V(f, \theta) \leq A_{\alpha}\|f\|_{\mathscr{M}_{\alpha}}$ for all $\theta$.

Proof. Suppose that $f \in \mathscr{M}_{\alpha}$ for some $\alpha>0$. If $|\xi|=1$ then there is a measure $\mu_{\xi}$ such that

$$
f(z) \frac{1}{(1-\xi z)^{\alpha}}=\int_{\Gamma} \frac{1}{(1-x z)^{\alpha}} d \mu_{\xi}(x) .
$$

Also, if $M=\|f\|_{\mathscr{M}_{\alpha}}$, and $\varepsilon>0$, then $\left\|\mu_{\xi}\right\| \leq M+\varepsilon$ for $|\xi|=1$.

It follows from (12) that

$$
f^{\prime}(z)=\alpha \int_{\Gamma} \frac{(1-\xi z)^{\alpha-1}(x-\xi)}{(1-x z)^{\alpha+1}} d \mu_{\xi}(x),
$$

and therefore

$$
\int_{0}^{1}\left|f^{\prime}(r \bar{\xi})\right| d r \leq \alpha \int_{\Gamma}\left[\int_{0}^{1} \frac{(1-r)^{\alpha-1}|x-\xi|}{|1-r x \bar{\xi}|^{\alpha+1}} d r\right] d\left|\mu_{\xi}\right|(x) .
$$

Let $I$ denote the inner integral on the right-hand side of (13). Because

$$
\begin{aligned}
|1-r x \bar{\xi}|^{\alpha+1} & =\left\{|1-r x \bar{\xi}|^{2}\right\}^{(\alpha+1) / 2}=\left\{(1-r)^{2}+r|1-x \bar{\xi}|^{2}\right\}^{(\alpha+1) / 2} \\
& \geq\left\{(1-r)^{2}+r^{2}|1-x \bar{\xi}|^{2}\right\}^{(\alpha+1) / 2}
\end{aligned}
$$

it follows that

$$
I \leq \int_{0}^{1} \frac{(1-r)^{\alpha-1} b}{\left\{(1-r)^{2}+r^{2} b^{2}\right\}^{(\alpha+1) / 2}} d r \equiv J,
$$

where $b=|1-x \bar{\xi}|$. The change of variables $y=r b /(1-r)$ shows that $J=\int_{0}^{\infty} \frac{1}{\left(1+y^{2}\right)^{(\alpha+1) / 2}} d y \equiv B_{\alpha}$. This integral converges since $\int_{1}^{\infty} 1 / y^{\beta} d y$ converges for $\beta>1$. Therefore (13) yields that

$$
\int_{0}^{1}\left|f^{\prime}(r \bar{\xi})\right| d r \leq \alpha \int_{\Gamma} B_{\alpha} d\left|\mu_{\xi}\right|(x) \leq A_{\alpha}(M+\varepsilon),
$$

where $A_{\alpha}=\alpha B_{\alpha}$. Let $\varepsilon \rightarrow 0$, the theorem is established.

Let $f \in \mathscr{M}_{\alpha}$. As a consequence of Theorem 2.6, the radial $\operatorname{limit} \lim _{r \rightarrow 1} f\left(r e^{i \theta}\right)$ exists for all $\theta$. Also, note that the conclusion of the theorem implies that $f$ is bounded. 
As an application of Theorem 2.6, we next give a number of simple examples of bounded functions which are not in $M_{\alpha}$ for any $\alpha>0$.

As a first example, let $f(z)=(1-z)^{-i}$, using the principal branch of the logarithm. Then $f$ is holomorphic in $\Delta$, and since $|f(z)|=e^{-\operatorname{Arg}(1-z)}$, it follows that $|f(z)|<e^{\pi / 2}$ for $|z|<1$. It is easy to verify that $f$ maps the interval $[0,1)$ onto the circle $\Gamma$ covered infinitely often and hence the curve $w=f(r), 0 \leq r<1$, is not rectifiable. It follows by Theorem 2.6 that $f \notin \mathscr{M}_{\alpha}$ for any $\alpha>0$.

In [9], it was shown that if $f$ is holomorphic in $\bar{\Delta}$, then $f \in \mathscr{M}_{\alpha}$ for all $\alpha>0$. In particular, this implies that a finite Blaschke product belongs to $\mathscr{M}_{\alpha}$ for $\alpha>0$. Theorem 2.5 provides a second proof of this fact, as follows. Let $I(z)=z$ for $|z|<1$. It is clear that $I \in \mathscr{M}_{\alpha}$ for $\alpha>0$. If $|\xi|<1$, then Theorem 2.5 implies that

$$
I\left(\frac{z+\xi}{1+\bar{\xi}_{z}}\right)=\frac{z+\xi}{1+\bar{\xi}_{z}} \in \mathscr{M}_{\alpha}, \quad \text { for } \alpha>0 .
$$

Since the finite product of functions in $\mathscr{M}_{\alpha}$ is itself in $\mathscr{M}_{\alpha}$, this proves the assertion.

We next show that there are infinite Blaschke products which are not in $\mathscr{M}_{\alpha}$ for any $\alpha>0$. Let $f(z)=\prod_{n=1}^{\infty}\left(a_{n}-z\right) /\left(1-a_{n} z\right)$ where $a_{n}=1-1 / 2^{n}$, $n=1,2, \ldots$. In [6] it was shown that there is a constant $A>0$ such that if $\rho_{n}=\frac{1}{2}\left(a_{n}+a_{n+1}\right)$ then $\left|f\left(\rho_{n}\right)\right| \geq A$ for $n=1,2, \ldots$. It follows that $\int_{0}^{1}\left|f^{\prime}(r)\right| d r=\infty$, so that by Theorem 2.6, $f \notin \mathscr{M}_{\alpha}$ for $\alpha>0$.

We note that in [10], it was proved that an inner function belongs to $\mathscr{M}_{1}$ if and only if it is a Blaschke product with the sequence of zeros satisfying the Frostman condition.

The next example shows that a function holomorphic in $\Delta$ and continuous in $\bar{\Delta}$ need not be in $\mathscr{M}_{\alpha}$ for any $\alpha>0$. In [17], L. Zalcman described a bounded region $D$ such that $\partial D$ is a Jordan curve, $z=1 \in \partial D$, and $z=1$ is not rectifiably accessible from the interior of $D$. Since $\partial D$ is a Jordan curve, any conformal mapping of $\Delta$ onto $D$ extends continuously to $\bar{\Delta}$. Let $f$ be such a map with $f(1)=1$. Then $f \notin \mathscr{M}_{\alpha}$, since the curve $w=f(r), 0 \leq r \leq 1$, is not rectifiable. The argument in [17] even shows that the power series for $f$ is uniformly convergent on $\partial \Delta$. Hence even with this additional condition we can still have $f \notin \mathscr{M}_{\alpha}$ for all $\alpha>0$.

The examples above give bounded functions for which the radial variation in one direction is infinite. A stronger result is presented in [14], where examples are given of infinite Blaschke products $B(z)$ for which the radial variation $V(B, \theta)=\infty$ for almost all $\theta$. Also, [14] includes the construction of a function $f$ holomorphic in $\Delta$ and continuous in $\bar{\Delta}$ for which $V(f, \theta)=\infty$ for almost all $\theta$.

In this section a condition is shown to be sufficient for membership in $\mathscr{M}_{\alpha}$ for every $\alpha>0$. Let $H^{1}$ denote the Hardy space of functions $f$ that are holomorphic in $\Delta$ and such that

$$
\sup _{0<r<1} \int_{0}^{2 \pi}\left|f\left(r e^{i \theta}\right)\right| d \theta<\infty .
$$


In [16, p. 20] it was proved by Vinogradov that if $f^{\prime} \in H^{1}$ then $f \in \mathscr{M}_{1}$. This result is generalized to $f^{\prime} \in H^{1}$ implies $f \in \mathscr{M}_{\alpha}$ for every $\alpha>0$. This strengthens the result in [9] which asserts that if $f$ is holomorphic in $\bar{\Delta}$ then $f \in \mathscr{M}_{\alpha}$ for every $\alpha>0$.

We begin by giving a new proof of Vinogradov's theorem. It may have independent interest especially since it shows that this result is related to the class of functions of bounded mean oscillation [5, p. 222]. Let $\mathscr{B}$ denote the set of functions $f$ holomorphic in $\Delta$ which can be expressed as $f=g+h$, where $g$ and $h$ are holomorphic in $\Delta, \operatorname{Re} g$ is bounded in $\Delta$, and $\operatorname{Im} h$ is bounded in $\Delta$. If $f \in \mathscr{B}$ then $\|f\|_{\mathscr{B}}$ is defined by $\inf \left(\|\operatorname{Re} g\|_{\infty}+\|\operatorname{Im} h\|_{\infty}\right)$ where $g$ and $h$ vary over all pairs as above. Here $\|u\|_{\infty}=\sup _{|z|<1}|u(z)|$ for any function $u$ defined in $\Delta$.

Lemma 3.1. Let $f$ be holomorphic in $\Delta$ and suppose that there is a holomorphic function $g$ and a constant $M>0$ such that

$$
|f(z)+g(\bar{z})| \leq M \quad \text { for }|z|<1 .
$$

Then $f \in \mathscr{B}$ and $\|f\|_{\mathscr{B}} \leq M$.

Proof. Let $s=\operatorname{Re} f, t=\operatorname{Im} f, u=\operatorname{Re} g$, and $v=\operatorname{Im} g$. The function $G$ defined by $G(z)=\frac{1}{2}[f(z)+\overline{g(\bar{z})}]$ is holomorphic in $\Delta$ and $\operatorname{Re} G(z)=$ $\frac{1}{2}[s(z)+u(\bar{z})]$. Hence (14) implies that $|\operatorname{Re} G(z)| \leq \frac{1}{2} M$ for $|z|<1$. The function $H$ defined by $H(z)=\frac{1}{2}[f(z)-\overline{g(\bar{z})}]$ is holomorphic in $\Delta$ and $\operatorname{Im} H(z)=\frac{1}{2}[t(z)+v(\bar{z})]$. Hence (14) implies $|\operatorname{Im} H(z)| \leq \frac{1}{2} M$ for $|z|<1$. Since $f=G+H$ this yields $f \in \mathscr{B}$. Moreover $\|f\|_{\mathscr{B}} \leq\|\operatorname{Re} G\|_{\infty}+$ $\|\operatorname{Im} H\|_{\infty} \leq M$.

Lemma 3.2. Let $f \in H^{\infty}$ and let $g$ be defined by

$$
g(z)=\frac{1}{z} \int_{0}^{z} \frac{f(w)}{1-w} d w
$$

for $|z|<1$. Then $\left|g^{\prime}(z)\right| \leq B\|f\|_{H^{\infty}} /|1-z|$ for $|z|<1$, where $B$ is an absolute constant.

Proof. We first show that if $|z|<1$ and $\alpha$ is the line segment from $w=0$ to $w=z$ then

$$
\int_{\alpha} \frac{1}{|1-w|^{2}}|d w| \leq \frac{\pi}{2} \frac{|z|}{|1-z|}
$$

This is clear if $z=0$. Also if $z$ is real and $z \neq 0$ then we have

$$
\int_{\alpha} \frac{1}{|1-w|^{2}}|d w|=|z| \int_{0}^{1} \frac{1}{(1-t z)^{2}} d t=\frac{|z|}{1-z},
$$

and hence (16) follows. Henceforth assume that $|z|<1$ and $z$ is not real. Then

$$
\begin{aligned}
\int_{\alpha} \frac{1}{|1-w|^{2}}|d w| & =|z| \int_{0}^{1} \frac{1}{(1-t z)(1-t \bar{z})} d t \\
& =\frac{|z|}{z-\bar{z}}\left\{\log \frac{1}{1-z}-\log \frac{1}{1-\bar{z}}\right\} \\
& =\frac{|z|}{z-\bar{z}} \int_{\beta} \frac{1}{1-w} d w
\end{aligned}
$$


where $\beta$ is the arc on the circle that is centered at $w=1$ and goes from $\bar{z}$ to $z$. Let $\theta$ denote the angle subtended by the arc $\beta$ and let $L$ denote the length of $\beta$. Then $|z-\bar{z}|=2|1-z| \sin (\theta / 2)$ and $L=|1-z| \theta$. Therefore

$$
\int_{\alpha} \frac{1}{|1-w|^{2}}|d w| \leq \frac{|z|}{|z-\bar{z}|} \frac{1}{|1-z|} L=\frac{\theta / 2}{\sin (\theta / 2)} \frac{|z|}{|1-z|} \leq \frac{\pi}{2} \frac{|z|}{|1-z|}
$$

since $0<\theta / 2 \leq \pi / 2$. This proves (16).

From (15) we obtain $z g^{\prime}(z)+g(z)=f(z) /(1-z)$. Hence an integration by parts yields

$$
\begin{aligned}
z^{2} g^{\prime}(z) & =\frac{z f(z)}{1-z}-z g(z)=\frac{z f(z)}{1-z}-\int_{0}^{z} \frac{f(w)}{1-w} d w \\
& =\frac{z f(z)}{1-z}-\frac{h(z)}{1-z}+\int_{0}^{z} \frac{h(w)}{(1-w)^{2}} d w
\end{aligned}
$$

where

$$
h(z)=\int_{0}^{z} f(w) d w
$$

for $|z|<1$. Clearly (17) implies $\|h\|_{H^{\infty}} \leq\|f\|_{H^{\infty}} \equiv M$. It follows that

$$
\left|z^{2} g^{\prime}(z)\right| \leq \frac{M}{|1-z|}+\frac{M}{|1-z|}+M \int_{\alpha} \frac{1}{|1-w|^{2}}|d w| \text {. }
$$

Therefore (16) implies that

$$
\left|z^{2} g^{\prime}(z)\right| \leq\left(2+\frac{\pi}{2}\right) M \frac{1}{|1-z|} \quad \text { for }|z|<1 .
$$

The function $G$ defined by $G(z)=(1-z) z^{2} g^{\prime}(z)$ is analytic in $\Delta$, has at least a second order zero at $z=0$ and satisfies $|G(z)| \leq B M$ for $|z|<1$ where $B=2+\pi / 2$. Hence $|G(z)| \leq B M|z|^{2}$ for $|z|<1$ and therefore $\left|g^{\prime}(z)\right| \leq B M /|1-z|$.

Lemma 3.3. Suppose that $f \in H^{\infty}$ and $g$ is defined by

$$
g(z)=\frac{1}{z} \int_{0}^{z} \frac{f(w)}{1-w} d w
$$

for $|z|<1$. Then $g \in \mathscr{B}$ and $\|g\|_{\mathscr{B}} \leq A\|f\|_{H^{\infty}}$ where $A$ is an absolute constant.

Proof. By equation (18) and Lemma 3.2, there is an absolute constant $B$ such that

$$
\left|g^{\prime}(z)\right| \leq \frac{B\|f\|_{H^{\infty}}}{|1-z|} \quad \text { for }|z|<1 .
$$

Let $|z|<1$ and let $\gamma$ denote the circle centered at 1 which passes through $z$ and has radius $r=|1-z|$. Let $\delta$ denote the subarc of $\gamma$ from $\bar{z}$ to $z$. Then

$$
g(z)-g(\bar{z})=\int_{\delta} g^{\prime}(w) d w
$$

and hence (19) implies that

$$
\left.|g(z)-g(\bar{z})| \leq \frac{B\|f\|_{H^{\infty}}}{r} \text { (length of } \delta\right) \leq \frac{\pi}{2} B\|f\|_{H^{\infty}} .
$$


An application of Lemma 3.1 in the special case where the functions there are related by $g=-f$ implies that $g \in \mathscr{B}$ and $\|g\|_{\mathscr{B}} \leq A\|f\|_{H^{\infty}}$ where $A=\pi B / 2$.

Lemma 3.4. Suppose that $f$ and $g$ are functions holomorphic in $\bar{\Delta}$ and let $F$ and $G$ be defined by

$$
F(z)=\frac{1}{1-z} \int_{z}^{1} f(w) d w
$$

and

$$
G(z)=\frac{1}{z} \int_{0}^{z} \frac{1}{1-w} g(w) d w
$$

Then

$$
\int_{0}^{2 \pi} f\left(e^{i \theta}\right) G\left(e^{-i \theta}\right) d \theta=\int_{0}^{2 \pi} F\left(e^{i \theta}\right) g\left(e^{-i \theta}\right) d \theta .
$$

Proof. There is a number $R>1$ such that $f(z)=\sum_{n=0}^{\infty} a_{n} z^{n}$ and $g(z)=$ $\sum_{n=0}^{\infty} b_{n} z^{n}$ for $|z|<R$. Then $F$ also is holomorphic in $\{z:|z|<R\}$ and $G$ is holomorphic in $\bar{\Delta}$ except possibly for a logarithmic singularity at $z=1$. In particular, $G \in H^{1}$ (in fact, $G \in H^{p}$ for all $p>0$ ). For $|z|<R$ we have

$$
\begin{aligned}
F(z) & =\frac{1}{1-z} \int_{z}^{1}\left(\sum_{n=0}^{\infty} a_{n} w^{n}\right) d w=\frac{1}{1-z} \sum_{n=0}^{\infty} \frac{a_{n}}{n+1}\left(1-z^{n+1}\right) \\
& =\sum_{n=0}^{\infty}\left\{\frac{a_{n}}{n+1} \sum_{k=0}^{n} z^{k}\right\}=\sum_{n=0}^{\infty}\left\{\sum_{k=n}^{\infty} \frac{a_{k}}{k+1}\right\} z^{n} .
\end{aligned}
$$

Therefore

$$
\int_{0}^{2 \pi} F\left(e^{i \theta}\right) g\left(e^{-i \theta}\right) d \theta=2 \pi \sum_{n=0}^{\infty}\left(\sum_{k=n}^{\infty} \frac{a_{k}}{k+1}\right) b_{n}=2 \pi \sum_{n=0}^{\infty}\left\{\frac{a_{n}}{n+1} \sum_{k=0}^{n} b_{k}\right\}
$$

For $|z|<1$, we have

$$
\begin{aligned}
G(z) & =\frac{1}{z} \int_{0}^{z}\left(\sum_{n=0}^{\infty} w^{n}\right)\left(\sum_{n=0}^{\infty} b_{n} w^{n}\right) d w \\
& =\frac{1}{z} \int_{0}^{z}\left(\sum_{n=0}^{\infty}\left(\sum_{k=0}^{n} b_{k}\right) w^{n}\right) d w \\
& =\sum_{n=0}^{\infty}\left(\frac{1}{n+1} \sum_{k=0}^{n} b_{k}\right) z^{n} .
\end{aligned}
$$

If $0<r<1$ then

$$
\int_{0}^{2 \pi} f\left(e^{i \theta}\right) G\left(r e^{-i \theta}\right) d \theta=2 \pi \sum_{n=0}^{\infty}\left(\frac{a_{n}}{n+1} \sum_{k=0}^{n} b_{k}\right) r^{n} \equiv H(r) .
$$

Since the series defining $H$ converges at $r=1$, Abel's theorem gives

$$
\lim _{r \rightarrow 1-} \int_{0}^{2 \pi} f\left(e^{i \theta}\right) G\left(r e^{-i \theta}\right) d \theta=\lim _{r \rightarrow 1-} H(r)=2 \pi \sum_{n=0}^{\infty}\left(\frac{a_{n}}{n+1} \sum_{k=0}^{n} b_{k}\right) .
$$


Also, because $f\left(e^{i \theta}\right)$ is bounded and $G \in H^{1}$ it follows that

$$
\lim _{r \rightarrow 1-} \int_{0}^{2 \pi} f\left(e^{i \theta}\right) G\left(r e^{-i \theta}\right) d \theta=\int_{0}^{2 \pi} f\left(e^{i \theta}\right) G\left(e^{-i \theta}\right) d \theta .
$$

Therefore by (23), (24), and (25),

$$
\int_{0}^{2 \pi} F\left(e^{i \theta}\right) g\left(e^{-i \theta}\right) d \theta=2 \pi \sum_{n=0}^{\infty}\left(\frac{a_{n}}{n+1} \sum_{k=0}^{n} b_{k}\right)=\int_{0}^{2 \pi} f\left(e^{i \theta}\right) G\left(e^{-i \theta}\right) d \theta .
$$

We thank D. J. Hallenbeck for pointing out and rectifying an error in our initial proof of Lemma 3.4.

Theorem C (Vinogradov). If $f^{\prime} \in H^{1}$, then $f \in \mathscr{M}_{1}$.

Proof. Suppose that $f^{\prime} \in H^{1}$ and $|\xi|=1$. We first note that

$$
\frac{f(z)}{\xi-z}=\frac{1}{\xi} \frac{f(z)}{1-\bar{\xi}_{z}} .
$$

Therefore by Lemma 2.1 , it is enough to show that $f(z) /(\xi-z) \in \mathscr{F}_{1}$, and that there is a constant $M>0$ such that $\|f(z) /(\xi-z)\|_{\mathscr{F}_{1}} \leq M$ for all $|\xi|=1$. Also note that

$$
\frac{f(z)}{\xi-z}=\frac{1}{\xi-z} \int_{0}^{z} f^{\prime}(w) d w+\frac{f(0)}{\xi-z} .
$$

Since $f(0) /(\xi-z) \in \mathscr{F}_{1}$ and since $\|f(0) /(\xi-z)\|_{\mathscr{F}_{1}}=|f(0)|$, it suffices to show that the function $(\xi-z)^{-1} \int_{0}^{z} f^{\prime}(w) d w$ belongs to $\mathscr{F}_{1}$ and that for some $M>0,\left\|(\xi-z)^{-1} \int_{0}^{z} f^{\prime}(w) d w\right\|_{\mathscr{F}_{1}} \leq M$ for all $|\xi|=1$. The argument is carried out with $\xi=1$ and a similar argument serves for all $\xi$ providing the same bound on the norm.

In our formulation we replace $f^{\prime}$ by $f$. In other words, assume that $f \in H^{1}$ and let

$$
g(z)=\frac{1}{1-z} \int_{0}^{z} f(w) d w \quad \text { for }|z|<1 .
$$

Then $g(z)=b /(1-z)-(1-z)^{-1} \int_{z}^{1} f(w) d w$, where $b=\int_{0}^{1} f(w) d w$.

First note that

$$
\begin{aligned}
|b| & \leq \int_{0}^{1}|f(w)||d w| \leq \int_{-1}^{1}|f(w) \| d w| \\
& \leq \frac{1}{2} \int_{0}^{2 \pi}\left|f\left(e^{i \theta}\right)\right| d \theta=\pi\|f\|_{H^{\prime}} \quad[4, \text { p. 46] }
\end{aligned}
$$

It follows that

$$
\left\|\frac{b}{1-z}\right\|_{\mathscr{F}_{1}} \leq \pi\|f\|_{H^{1}}
$$

Next let $k(z)=(1-z)^{-1} \int_{z}^{1} f(w) d w$. Let $A$ denote the space of functions holomorphic in $\Delta$ and continuous in $\bar{\Delta}$. To show that $k \in \mathscr{F}_{1}$ it suffices to prove that there is a constant $A>0$ such that

$$
\left|\int_{0}^{2 \pi} k\left(r e^{i \theta}\right) h\left(e^{-i \theta}\right) d \theta\right| \leq A\|h\|_{H^{\infty}}
$$


for $0<r<1$ and for all $h \in A$. This inequality will be obtained where $A=B\|f\|_{H^{1}}$ and $B$ is an absolute constant. This will imply that

$$
\|k\|_{\mathscr{F}_{1}} \leq B\|f\|_{H^{1}}
$$

and it then follows from (26), (27), and (29) that $\|g\|_{\mathscr{F}_{1}} \leq(\pi+B)\|f\|_{H^{1}}$.

By first making the change of variables $z \rightarrow \rho z$ where $0<\rho<1$ and then letting $\rho \rightarrow 1$, we may assume that $f$ and $h$ are holomorphic in $\bar{\Delta}$. Then $k$ is holomorphic in $\bar{\Delta}$. We now show that it suffices to prove that

$$
\left|\int_{0}^{2 \pi} k\left(e^{i \theta}\right) h\left(e^{-i \theta}\right) d \theta\right| \leq C\|f\|_{H^{1}}\|h\|_{H^{\infty}},
$$

where $C$ is an absolute constant. For $0 \leq r \leq 1$ let $F(r)=\int_{0}^{2 \pi} k\left(r e^{i \theta}\right) h\left(e^{i \theta}\right) d \theta$. Assuming(30) we get $|F(1)| \leq C\|f\|_{H^{1}}\|h\|_{H^{\infty}}$. Since $F$ is continuous in $[0,1]$, there exists $r_{0} \quad\left(0<r_{0}<1\right)$ such that $|F(r)| \leq 2|F(1)|$ for $r_{0} \leq r \leq 1$. Therefore

$$
|F(r)| \leq 2 C\|f\|_{H^{1}}\|h\|_{H^{\infty}} \quad \text { for } r_{0} \leq r<1 .
$$

Suppose now that $0 \leq r \leq r_{0}$. Then

$$
|F(r)| \leq \int_{0}^{2 \pi}\left|k\left(r e^{i \theta}\right)\right|\left|h\left(e^{-i \theta}\right)\right| d \theta \leq\|h\|_{H^{\infty}} \int_{0}^{2 \pi}\left|k\left(r_{0} e^{i \theta}\right)\right| d \theta .
$$

Without loss of generality we may assume that $f \neq 0$. Then $k \neq 0,\|f\|_{H^{1}}>0$, and $\int_{0}^{2 \pi}\left|k\left(r_{0} e^{i \theta}\right)\right| d \theta>0$. Therefore for some $D>0, \int_{0}^{2 \pi}\left|k\left(r_{0} e^{i \theta}\right)\right| d \theta=$ $D\|f\|_{H^{1}}$. It follows that

$$
|F(r)| \leq D\|f\|_{H^{1}}\|h\|_{H^{\infty}} \quad \text { for } 0 \leq r \leq r_{0} .
$$

Letting $B=\max (2 C, D)$, relations (31) and (32) imply that

$$
|F(r)| \leq B\|f\|_{H^{1}}\|h\|_{H^{\infty}} \quad \text { for } 0 \leq r \leq 1 .
$$

This proves (28).

It remains to prove the assertion (30). Let $m(z)=z^{-1} \int_{0}^{z}(1-w)^{-1} h(w) d w$. Lemma 3.3 implies that $m \in \mathscr{B}$ and $\|m\|_{\mathscr{B}} \leq C\|h\|_{H^{\infty}}$ for an absolute constant $C$. We have $m=p+q$ where $p$ and $q$ are holomorphic in $\Delta$ and $u=\operatorname{Re} p$ and $v=\operatorname{Im} q$ are bounded and $\|u\|_{\infty}+\|v\|_{\infty} \leq C\|h\|_{H^{\infty}}$. Now

$$
\int_{0}^{2 \pi} f\left(e^{i \theta}\right) m\left(e^{-i \theta}\right) d \theta=\int_{0}^{2 \pi} f\left(e^{i \theta}\right) p\left(e^{-i \theta}\right) d \theta+\int_{0}^{2 \pi} f\left(e^{i \theta}\right) q\left(e^{-i \theta}\right) d \theta .
$$

Using power series and the orthonormal relations for the trigonometric functions, this equals

$$
\int_{0}^{2 \pi} f\left(e^{i \theta}\right) u\left(e^{-i \theta}\right) d \theta+i \int_{0}^{2 \pi} f\left(e^{i \theta}\right) v\left(e^{-i \theta}\right) d \theta
$$

Hence

$$
\begin{aligned}
\left|\int_{0}^{2 \pi} f\left(e^{i \theta}\right) m\left(e^{-i \theta}\right) d \theta\right| & \leq\|u\|_{\infty}\|f\|_{H^{1}}+\|v\|_{\infty}\|f\|_{H^{1}} \\
& =\left(\|u\|_{\infty}+\|v\|_{\infty}\right)\|f\|_{H^{1}} \\
& \leq C\|f\|_{H^{1}}\|h\|_{H^{\infty}}
\end{aligned}
$$


Because of Lemma 3.4, this yields

$$
\left|\int_{0}^{2 \pi} k\left(e^{i \theta}\right) h\left(e^{-i \theta}\right) d \theta\right| \leq C\|f\|_{H^{1}}\|h\|_{H^{\infty}},
$$

which is the required inequality.

The argument used to prove Theorem $\mathrm{C}$ does not depend on the duality theorem about $H^{1}$ and BMO proved by C. Fefferman [5, p. 245]. It is interesting to note that the function $g$ defined in Lemma 3.3 can be shown to have bounded mean oscillation by a fairly direct argument.

The essential ideas for proving Theorem $\mathrm{C}$ as developed above are due to Boris Korenblum [12]. The authors would like to thank Korenblum for several helpful conversations about multipliers.

Theorem 3.5. If $f^{\prime} \in H^{1}$, then $f \in \mathscr{M}_{\alpha}$ for all $\alpha>0$.

Proof. Let $f^{\prime} \in H^{1}$. By Theorem C, $f \in \mathscr{M}_{1}$, and by Theorem 2.2 it follows that $f \in \mathscr{M}_{\alpha}$ for every $\alpha>1$.

In the case $0<\alpha<1$, let $g \in \mathscr{F}_{\alpha}$, and let $h=f g$. By Theorem A, it suffices to show that $h^{\prime} \in \mathscr{F}_{\alpha+1}$.

Since $g \in \mathscr{F}_{\alpha}$, Theorem A implies that $g^{\prime} \in \mathscr{F}_{\alpha+1}$. By the previous part of the proof, $f \in \mathscr{M}_{\alpha+1}$, and therefore

$$
f g^{\prime} \in \mathscr{F}_{\alpha+1} \text {. }
$$

Because $f^{\prime} \in H^{1}$, it follows that $f^{\prime} \in \mathscr{F}_{1}\left[4\right.$, p. 34]. By assumption, $g \in \mathscr{F}_{\alpha}$ and so Theorem B implies that

$$
f^{\prime} g \in \mathscr{F}_{\alpha+1} \text {. }
$$

Since $h^{\prime}=f g^{\prime}+f^{\prime} g,(33)$ and (34) show that $h^{\prime} \in \mathscr{F}_{\alpha+1}$, or equivalently, $h \in \mathscr{F}_{\alpha}$. This proves that $f \in \mathscr{M}_{\alpha}$ for $0<\alpha<1$.

Theorem 3.5 is sharp, since there are functions $f$ such that $f^{\prime} \in H^{p}$ $(0<p<1)$ and $f$ is not bounded. By Theorem 2.3, such functions are not multipliers.

One example where Theorem 3.5 applies concerns bounded convex maps. Suppose that $f$ is holomorphic in $\Delta$ and that $f$ maps $\Delta$ one-to-one onto a bounded convex region. Since the boundary $C$ of such a region is rectifiable and since $C$ is a Jordan curve, it follows that $f^{\prime} \in H^{1}[4$, p. 44]. Therefore, $f \in \mathscr{M}_{\alpha}$, for $\alpha>0$.

\section{4}

Suppose that $f(z)=\sum_{n=0}^{\infty} a_{n} z^{n}$ is holomorphic in $\Delta$. Let

$$
s_{n}(z)=\sum_{j=0}^{n} a_{j} z^{j}
$$

and

$$
\sigma_{n}(z)=\frac{1}{n+1} \sum_{j=0}^{n} s_{j}(z) .
$$

By a classical result [3, p. 439], the function $f$ is bounded if and only if the sequence $\sigma_{n}(z)$ is uniformly bounded for $n=0,1, \ldots$ and for $|z| \leq 1$, and 
in this case, $\|f\|_{H^{\infty}}=\sup \left\{\left\|\sigma_{n}\right\|_{H^{\infty}}: n=0,1, \ldots\right\}$. This result is generalized in this section, in terms of polynomials which are generated in the study of the multiplier problem.

Definition. For $f(z)=\sum_{n=0}^{\infty} a_{n} z^{n} \quad(|z|<1)$, let $P_{n}(z ; \alpha)=\frac{1}{A_{n}(\alpha)}\left\{A_{n}(\alpha) a_{0}+A_{n-1}(\alpha) a_{1} z+\cdots+A_{1}(\alpha) a_{n-1} z^{n-1}+A_{0}(\alpha) a_{n} z^{n}\right\}$ where $\alpha>0, n=0,1, \ldots$, and $z \in \mathbf{C}$.

Theorem 4.1. If $f \in \mathscr{M}_{\alpha}$, then $\left\|P_{n}(z ; \alpha)\right\|_{H^{\infty}} \leq\|f\|_{\mathscr{M}_{\alpha}}$ for $n=0,1, \ldots$. Proof. Let $f \in \mathscr{M}_{\alpha}$ and suppose that $M>\|f\|_{\mathscr{M}_{\alpha}}$. If $|x|=1$ then we have $f(z) /(1-x z)^{\alpha} \in \mathscr{F}_{\alpha}$. Also,

$$
\left\|f(z) \frac{1}{(1-x z)^{\alpha}}\right\|_{\mathscr{F}_{\alpha}} \leq M \quad \text { for all }|x|=1 .
$$

Therefore for each $x \quad(|x|=1)$ there is a measure $\mu_{x} \in \mathscr{M}$ such that

$$
f(z) \frac{1}{(1-x z)^{\alpha}}=\int_{\Gamma} \frac{1}{(1-y z)^{\alpha}} d \mu_{x}(y),
$$

and $\left\|\mu_{x}\right\| \leq M$ for $|x|=1$.

If $f(z)=\sum_{n=0}^{\alpha} a_{n} z^{n}$, then $f(z) /(1-x z)^{\alpha}=\sum_{n=0}^{\infty} b_{n} z^{n}$ where

$$
b_{n}=A_{0}(\alpha) a_{n}+A_{1}(\alpha) a_{n-1} x+\cdots+A_{n-1}(\alpha) a_{1} x^{n-1}+A_{n}(\alpha) a_{0} x^{n} .
$$

If

$$
\int_{\Gamma} \frac{1}{(1-y z)^{\alpha}} d \mu_{x}(y)=\sum_{n=0}^{\infty} c_{n} z^{n}
$$

then

$$
c_{n}=A_{n}(\alpha) \int_{\Gamma} y^{n} d \mu_{x}(y) .
$$

Because of (35), $b_{n}=c_{n}$, or

$$
x^{n} P_{n}\left(\frac{1}{x} ; \alpha\right)=\int_{\Gamma} y^{n} d \mu_{x}(y) .
$$

Since $\left\|\mu_{x}\right\| \leq M$ for $|x|=1$, (36) implies that $\left|P_{n}(1 / x ; \alpha)\right| \leq M$ for $|x|=1$ and $n=0,1, \ldots$. Equivalently $\left|P_{n}(z ; \alpha)\right| \leq M$ for $|z|=1$ and hence $\left\|P_{n}(z ; \alpha)\right\|_{H^{\infty}} \leq M$. Since this holds for every $M>\|f\|_{\mathscr{K}_{\alpha}}$, this proves the theorem.

The next results generalize the statement made previously concerning the Cesàro sums $\sigma_{n}(z)$ for a function holomorphic in $\Delta$. Note that $\sigma_{n}(z)=$ $P_{n}(z ; 2)$ since the binomial coefficient $A_{n}(2)=n+1$ for $n=0,1, \ldots$.

Theorem 4.2. Suppose that $f$ is holomorphic in $\Delta$ and that $\left|P_{n}(z ; \alpha)\right| \leq M$ for $|z| \leq 1$ and $n=0,1, \ldots$. Then $f \in H^{\infty}$ and $\|f\|_{H^{\infty}} \leq M$. 
Proof. Let $f(z)=\sum_{n=0}^{\infty} a_{n} z^{n}$ for $|z|<1$. Assume that $0 \leq r<1$ and $|x|=1$. Then

$$
\begin{aligned}
\frac{1}{(1-r)^{\alpha}} f(r x) & =\left\{\sum_{n=0}^{\infty} A_{n}(\alpha) r^{n}\right\}\left\{\sum_{n=0}^{\infty} a_{n} r^{n} x^{n}\right\} \\
& =\sum_{n=0}^{\infty}\left(\sum_{k=0}^{n} A_{n-k}(\alpha) a_{k} x^{k}\right) r^{n} \\
& =\sum_{n=0}^{\infty} A_{n}(\alpha) P_{n}(x ; \alpha) r^{n}
\end{aligned}
$$

Therefore

$$
\begin{aligned}
\frac{1}{(1-r)^{\alpha}}|f(r x)| & \leq \sum_{n=0}^{\infty} A_{n}(\alpha)\left|P_{n}(x ; \alpha)\right| r^{n} \\
& \leq M \sum_{n=0}^{\infty} A_{n}(\alpha) r^{n}=M \frac{1}{(1-r)^{\alpha}}
\end{aligned}
$$

and so $|f(r x)| \leq M$. Since this holds for all $r$ and $x$, it follows that $|f(z)| \leq$ $M$ for $|z|<1$.

The following lemma will be used to establish a partial converse to Theorem 4.2. The kernels $T_{n}(\theta ; \alpha)$ introduced in the lemma are well known, and are studied in [18].

Lemma 4.3. Let $\mu_{0}=\frac{1}{2}$ and for $k=1,2, \ldots$ let $\mu_{k}(\theta)=\cos k \theta$. Also let

$$
T_{n}(\theta ; \alpha)=\frac{1}{A_{n}(\alpha)} \sum_{k=0}^{n} A_{n-k}(\alpha) \mu_{k}(\theta) \text {. }
$$

(a) If $\alpha \geq 2$ then $T_{n}(\theta ; \alpha) \geq 0$ for $0 \leq \theta \leq 2 \pi$ and $n=0,1, \ldots$.

(b) If $1<\alpha<2$ there is a constant $B(\alpha)$ such that

$$
\frac{1}{2 \pi} \int_{0}^{2 \pi}\left|T_{n}(\theta ; \alpha)\right| d \theta \leq B(\alpha) \quad \text { for } n=0,1, \ldots
$$

Proof. First consider the case $\alpha=2$. Then (a) is a known fact and the argument for it is as follows. Since $A_{n}(2)=n+1$ for $n=0,1, \ldots$,

$$
\begin{aligned}
T_{n}(\theta ; 2) & =\frac{1}{n+1}\left\{\frac{n+1}{2}+\sum_{k=1}^{n}(n-k+1) \cos k \theta\right\} \\
& =\frac{1}{2} \sum_{k=-n}^{n}\left(1-\frac{|k|}{n+1}\right) e^{i k t}=\frac{1}{2} \frac{1}{n+1}\left\{\frac{\sin \frac{n+1}{2} \theta}{\sin \frac{1}{2} \theta}\right\}^{2} \geq 0
\end{aligned}
$$

This proves (a) when $\alpha=2$. 
Suppose that $\alpha>0$ and $\beta>0$. Then

$$
\begin{aligned}
\sum_{n=0}^{\infty} A_{n}(\alpha+\beta) z^{n} & =\frac{1}{(1-z)^{\alpha+\beta}}=\frac{1}{(1-z)^{\alpha}} \frac{1}{(1-z)^{\beta}} \\
& =\sum_{n=0}^{\infty} A_{n}(\alpha) z^{n} \sum_{n=0}^{\infty} A_{n}(\beta) z^{n} \\
& =\sum_{n=0}^{\infty}\left\{\sum_{k=0}^{n} A_{n-k}(\alpha) A_{k}(\beta)\right\} z^{n} .
\end{aligned}
$$

This shows that

$$
A_{n}(\alpha+\beta)=\sum_{k=0}^{n} A_{n-k}(\alpha) A_{k}(\beta) .
$$

Now assume that $\alpha>2$. From (37), it follows that

$$
\begin{aligned}
A_{n}(\alpha) T_{n}(\theta ; \alpha) & =\sum_{k=0}^{n} A_{n-k}(\alpha) \mu_{k}(\theta) \\
& =\sum_{k=0}^{n}\left\{\sum_{j=0}^{n-k} A_{n-k-j}(2) A_{j}(\alpha-2)\right\} \mu_{k}(\theta) \\
& =\sum_{j=0}^{n}\left\{\sum_{k=0}^{n-j} A_{n-j-k}(2) \mu_{k}(\theta)\right\} A_{j}(\alpha-2) \\
& =\sum_{j=0}^{n} T_{n-j}(\theta ; 2) A_{n-j}(2) A_{j}(\alpha-2) .
\end{aligned}
$$

Because $A_{n-j}(2)>0, A_{j}(\alpha-2)>0$, and $T_{n-j}(\theta ; 2) \geq 0$, this implies that $A_{n}(\alpha) T_{n}(\theta ; \alpha) \geq 0$. This proves (a) for $\alpha>2$.

A proof of $(b)$ is contained in $[18$, Vol. 1, p. 94], where it is shown that the kernel

$$
K_{n}^{\beta}(\theta)=\frac{1}{A_{n}(\beta+1)} \sum_{k=0}^{n} A_{n-k}(\beta) D_{k}(\theta)
$$

is "quasipositive" for $0<\beta<1$. Here $D_{k}(\theta)$ denotes the Dirichlet kernel $\frac{1}{2} \sum_{j=-k}^{k} e^{i j \theta}$. Note that $K_{n}^{\alpha-1}(\theta)=T_{n}(\theta ; \alpha)$, and since $1<\alpha<2$ by assumption, this establishes (b).

The authors would like to thank B. Muckenhoupt, who provided the proof of (a) for $\alpha>2$, and who pointed out that this fact is known.

Theorem 4.4. For each $\alpha>1$ there is a constant $C(\alpha)$ such that if $f \in H^{\infty}$, then

$$
\left\|P_{n}(z ; \alpha)\right\|_{H^{\infty}} \leq C(\alpha)\|f\|_{H^{\infty}},
$$

for $n=0,1, \ldots$. When $\alpha \geq 2$, (38) holds with $C(\alpha)=1$.

Proof. The orthonormal relations for the trigonometric functions imply that

$$
\frac{1}{2 \pi} \int_{0}^{2 \pi} f\left(z e^{i \theta}\right) T_{n}(\theta ; \alpha) d \theta=\frac{1}{2} P_{n}(z ; \alpha)
$$

for $|z|<1$. 
Suppose that $\alpha \geq 2,|z|<1$, and $f \in H^{\infty}$. Then (39) and (a) in Lemma 4.3 imply that

$$
\frac{1}{2}\left|P_{n}(z ; \alpha)\right| \leq \frac{1}{2 \pi} \int_{0}^{2 \pi}\|f\|_{H^{\infty}} T_{n}(\theta ; \alpha) d \theta=\frac{1}{2}\|f\|_{H^{\infty}} .
$$

This proves the theorem in the case $\alpha \geq 2$.

Now suppose that $1<\alpha<2,|z|<1$, and $f \in H^{\infty}$. Then (39) and (b) in Lemma 4.3 imply that

$$
\frac{1}{2}\left|P_{n}(z ; \alpha)\right| \leq\|f\|_{H^{\infty}} \frac{1}{2 \pi} \int_{0}^{2 \pi}\left|T_{n}(\theta ; \alpha)\right| d \theta \leq B(\alpha)\|f\|_{H^{\infty}} .
$$

This proves the theorem where $C(\alpha)=2 B(\alpha)$.

The assertion in Theorem 4.4 does not hold for $\alpha=1$. This is because there are functions bounded and holomorphic in $\Delta$ such that the sequence of partial sums $s_{n}$ is not uniformly bounded in $\Delta[3$, p. 444]. Also note that $P_{n}(z ; 1)=s_{n}(z)$.

\section{BIBLIOGRAPHY}

1. P. Bourdon and J. A. Cima, On integrals of Cauchy-Stieltjes type, Houston J. Math. 14 (1988), 465-474.

2. L. Brickman, D. J. Hallenbeck, T. H. MacGregor, and D. R. Wilken, Convex hulls and extreme points of families of starlike and convex mappings, Trans. Amer. Math. Soc. 185 (1973), 413-428.

3. P. Dienes, The Taylor series, Oxford Univ. Press, London, 1931.

4. P. L. Duren, Theory of $H^{p}$ spaces, Academic Press, New York, 1970.

5. J. B. Garnett, Bounded analytic functions, Academic Press, New York, 1981.

6. D. J. Hallenbeck and T. H. MacGregor, Radial growth and variation of bounded analytic functions, Proc. Edinburgh Math. Soc. (2) 31 (1988), 489-498.

7. V. P. Havin, On analytic functions representable by an integral of Cauchy-Stieltjes type, Vestnik Leningrad Univ. 13 (1958), no. 1 (Ser. Mat. Meh. Astronom. vyp. 1), 66-79. (Russian)

8. _ Relations between certain classes of functions regular in the unit disk, Vestnik Leningrad Univ. 17 (1962), no. 1 (Ser. Mat. Meh. Astronom. vyp. 1), 102-110. (Russian)

9. R. A. Hibschweiler and T. H. MacGregor, Closure properties of families of Cauchy-Stieltjes transforms, Proc. Amer. Math. Soc. 105 (1989), 615-621.

10. S. V. Hruščev and S. A. Vinogradov, Inner functions and multipliers of Cauchy type integrals, Ark. Mat. 19 (1981), 23-42.

11. P. Koosis, Introduction to $H_{p}$ spaces, Cambridge Univ. Press, Cambridge, 1980.

12. B. Korenblum, private communication.

13. T. H. MacGregor, Analytic and univalent functions with integral representations involving complex measures, Indiana Univ. Math. J. 36 (1987), 109-130.

14. W. Rudin, The radial variation of analytic functions, Duke Math. J. 22 (1955), 235-242.

15. S. A. Vinogradov, M. G. Goluzina, and V. P. Havin, Multipliers and divisors of CauchyStieltjes integrals, Zap. Naučn. Sem. Leningrad. Otdel. Mat. Inst. Steklov. (LOMI) 19 (1970), 55-78. (Russian)

16. S. A. Vinogradov, Properties of multipliers of Cauchy-Stieltjes integrals and some factorization problems for analytic functions, Amer. Math. Soc. Transl. (2) 115 (1980), 1-32. 
17. L. Zalcman, Real proofs of complex theorems (and vice versa), Amer. Math. Monthly 81 (1974), 115-137.

18. A. Zygmund, Trigonometric series, Cambridge Univ. Press, Cambridge, 1968.

Department of Mathematics, University of New Hampshire, Durham, New Hampshire 03824

Department of Mathematics, State University of New York at Albany, Albany, New YORK 12222 